\title{
Análisis mutifrecuencia de la reflectividad del fondo marino para explorar la distribución superficial de sedimentos marinos al suroeste de Islas Hormigas de Afuera, costa afuera de Callao
}

\section{Seafloor reflectivity mutifrequency analysis to explore the surficial distribution of marine sediments to the southeast of Hormigas Islands, offshore Callao}

\author{
Gery Herbozo ${ }^{1}$, Julia Fuertes ${ }^{1}$, David Mamani ${ }^{1}$, Federico Velazco ${ }^{2}$
}

${ }^{1}$ Área Funcional de Hidroacústica (AFH), Dirección General de Investigaciones en Hidroacústica, Sensoramiento Remoto y Artes de Pesca (DGIHSA), Instituto del Mar del Perú (IMARPE)

2 Área de Geología Marina (AGM), Dirección General de Investigaciones Oceanográficas y Cambio Climático

(DGIOCC), Instituto del Mar del Perú (IMARPE)

DOI: https://doi.org/10.33017/RevECIPeru2016.0005/

\section{Resumen}

El conocimiento de la naturaleza sedimentaria del fondo marino es un aspecto importante para inferir la distribución de recursos pesqueros demersales de importancia económica (e.g, la merluza peruana (Merluccius gayi peruanus)), la localización de zonas propicias para el desarrollo de actividades de acuicultura, el estudio de procesos biogeoquímicos en la columna de agua y la estimación de abundancia de organismos bentónicos localizados sobre el fondo marino (e.g., Thioploca). El objetivo de este estudio es inferir la composición general del primer metro de sedimentos marinos localizados al suroeste de las Islas Hormigas de Afuera, costa afuera de Callao mediante información de retrodispersión acústica derivada de un sistema hidroacústico multifrecuencia (ecosonda científica EK60 de 5 frecuencias, $200 \mathrm{kHz}, 120 \mathrm{kHz}, 70 \mathrm{kHz}$, $38 \mathrm{kHz}$ y $18 \mathrm{kHz}$ ) del Instituto del Mar del Perú (IMARPE) utilizado para realizar evaluaciones pesqueras. En este estudio se obtuvo información de retrodispersión ( $S v$ mean) del primer metro por debajo del fondo marino a lo largo de un transecto de $7.5 \mathrm{~km}$ en la plataforma continental (160 m de columna de agua). Esta información fue utilizada para analizar la reflectividad de los sedimentos marinos investigados. Adicionalmente, se compiló información sedimentológica del fondo marino para comparar los resultados obtenidos de reflectividad. Como resultado, se determinó que existen seis patrones de reflectividad que definirían ciertos tipos de sedimentos superficiales. Estos patrones, sin embargo, no pudieron ser corroborados dado que no se encontró información sedimentológica a lo largo del transecto analizado. La reflectividad en tres de los patrones elegidos (p2, p4 y p5) podrían representar zonas donde existen sustratos duros cerca al fondo marino (e.g., cortezas de fosforita y/o rocas del orden de $\mathrm{cm}$ de espesor) con sedimentos finos y gruesos (e.g., fangos y arenas gruesas) por debajo de estos sustratos duros. Debido a la permeabilidad acústica de una zona de la capa superficial investigada, uno de los patrones observados (p3) podría representar sedimentos finos asociados al mud lens. Este tipo de depocentros sedimentarios estan compuestos por sedimentos finamente laminados que son permeables a la energía acústica (e.g., arcillas y/o una intercalación de sustratos finos). Los dos patrones localizados a los extremos del transecto (p1 y p6) podrían representar zonas donde existen frentes de gas natural distribuidos en sedimento fangoso. De esta 
investigación concluimos que no es posible clasificar los tipos de sedimentos basados únicamente en información de reflectividad. Sin embargo, la interrelación de reflectividades basada en información multifrecuencia si permite observar patrones, los cuales necesitan ser calibrados con muestras de sedimentos in-situ para definir una clasificación en zonas donde solamente existe información hidroacústica.

\section{Descriptores: Perú, hidroacústica, multifrecuencia, fondo marino, sedimentos marinos superficiales}

\section{Abstract}

The study of the sedimentary nature of the seafloor is a key aspect to infer the spatial distribution of demersal fisheries resources (e.g., hake (Merluccius gayi peruanus)), to locate adequate zones for aquaculture farms, to examine biogeochemical processes occurred in the water column and to estimate the abundance of benthic organisms lying at the seafloor (e.g., Thioploca). The aim of this study is to infer, using acoustic backscattering mutifrequency information from an EK60 scientific echosounder (200 kHz, $120 \mathrm{kHz}, 70 \mathrm{kHz}, 38 \mathrm{kHz}, 18 \mathrm{kHz}$ ), the bulk composition of the first meter of marine sediments located to the southeast of Hormigas de Afuera Islands, offshore Callao. This hydroacoustic multifrequency system is routinely used for the assessment of fisheries resources for the Peruvian Sea Institute (IMARPE). In this study we used backscattering information (Sv mean) from the first meter below the sea bottom along a $7.5-\mathrm{km}$ transect in the shelf (160 m water depth). This information represents the acoustic reflectivity of the investigated sediments. In addition, sediment sampling information from the seafloor was compiled to compare results from the reflectivity analyses. As result, we defined that there are six reflectivity patterns that would define certain types of surficial sediments. However, these patterns could not be compared to in-situ sediment samples because we found no historical sediment information along the studied transect. Reflectivity of the surficial sediment layer from three of the chosen patterns (p2, p4 and p5) may represent seafloor zones with hard substrata near the sea bottom (e.g., phosphorite crusts and/or small rocks of few $\mathrm{cm}$ thick) overlying fine- and coarsed-grained sediments (e.g., mud and coarse sands). Due to the acoustic permeability of one zone of this surficial layer, one of the observed patterns (p3) may represent fine-grained sediments associated to the mud lens. This type of sediment depocenters are composed by fine-grained, laminated sediments that are permeable to the acoustic energy (e.g., clays and/or a mix of fine-grained sediments). The two last patterns ( $p 1$ and p6), located at the northwest and southeast ends of the transect, may define zones with gas fronts in mud sediments. We conclude that it is not possible to classify sediment types based unambiguously on reflectivity information. However, the use of multifrequency reflectivity information does allow the characterization of sediment patterns. Such information needs necessarily to be calibrated with in-situ sediment samples in order to define the classification of marine surface sediment types at the seafloor where only hydroacoustic information is present.

\section{Keywords: Peru, hydroacoustics, mutifrequency, seafloor, surficial marine sediments}

\section{Introducción}

Los sedimentos marinos superficiales a lo largo de la plataforma continental del mar peruano se caracterizan por tener altos contenidos de carbono orgánico producto de la alta productividad primaria y el perenne proceso de afloramiento costero presente a lo largo de la costa Peruana [e.g., Morón, 2000; D'Hondt et al., 2003; Böning et al., 2004]. En estas zonas del margen continental peruano, localizadas dentro de la Zona Mínima de
Oxígeno (ZMO) [Stramma et al., 2008]; se han acumulado sedimentos anóxicos que estan caracterizados por estructuras geológicas (e.g., laminaciones de 1 milímetro de espesor) que han servido para realizar reconstrucciones paleoceanográficas de alta resolución [Reinhardt et al., 2002; Gutiérrez et al., 2006]. Estos depósitos laminados son conocidos como mud lens [Suess and von Huene, 1988; Reinhardt et al., 2002; Salvatteci et al., 2014] y están compuestos principalmente por sedimentos biogénicos de 
textura fina (e.g., arcillas). En estos depósitos, Gutiérrez et al. [2006] han recuperado muestras de hasta 1.5 metros de sedimento finamente laminado, mientras que Reinhardt et al. [2002], Suess and von Huene [1988] y D'Hondt et al. [2003] han recuperado muestras con espesores mayores a 2 metros. Investigaciones paleoceanográficas en estos registros sedimentarios han permitido reconstruir fluctuaciones locales y regionales sobre la interacción entre El Niño, La Niña, la intensidad del afloramiento costero y la abundancia de especies pelágicas tales como la anchoveta peruana durante los últimos 20000 años [e.g., Rein et al., 2005; Gutiérrez et al., 2009; Salvatteci et al., 2013]. Debido a la textura de la cobertura superficial de los mud lens, Gutiérrez and Herbozo [2004] estimaron en base a información de retrodispersión acústica de una ecosonda científica monofrecuencia $(38 \mathrm{kHz}$ ) del Instituto del Mar del Perú (IMARPE) que es posible mapear tales depósitos debido a la reflectividad acústica del material sedimentario presente. Estos autores además plantearon que es posible clasificar de forma exploratoria la presencia de otro tipo de sustratos (e.g., rocas) de acuerdo a la "dureza" acústica del fondo marino. Tal identificación remota de sustratos es clave para ubicar los mud lens, ya que estos depósitos presentan una distribución espacial heterogénea a lo largo de la plataforma y el talud continental [e.g., Suess and von Huene, 1988; Reinhardt et al., 2002; Salvatteci et al., 2014]. En los últimos años, IMARPE ha implementado un sistema multifrecuencia en sus buques de investigación científica para la evaluación de recursos pesqueros (i.e., BIC José Olaya Balandra y BIC Luis Flores Portugal). Para el caso del fondo marino, este sistema permitiría una caracterización más detallada de los sustratos superficiales ya que la reflectividad acústica es dependiente de las frecuencias utilizadas. Este aspecto ha generado prometedores resultados en la diferenciación de habitats en zonas costeras [e.g., Cuff et al., 2015]. El objetivo de este estudio es investigar la reflectividad acústica de la cobertura sedimentaria superficial del fondo marino en una zona al suroeste de Islas Hormigas de Afuera (Figura 1) utilizando información hidroacústica multifrecuencia adquirida como parte de un experimento de intercalibraciones usando el BIC José Olaya Balandra [Herbozo et al., 2016c].

\section{Metodología}

Para realizar el análisis de reflectividad acústica del primer metro de sedimentos marinos, se utilizó información de volumen medio de retrodispersión acústica (Mean Volumen Backscattering Strength ó Sv mean) adquirida con el sistema multifrecuencia del ecosonda científica SIMRAD EK60 [Andersen, 2001] localizado en el BIC José Olaya Balandra del IMARPE. Este sistema splitbeam esta compuesto de transductores acústicos de geometría circular y utiliza cinco frecuencias $(200 \mathrm{kHz}, 120 \mathrm{kHz}, 70$ $\mathrm{kHz}, 38 \mathrm{kHz}$ y $18 \mathrm{kHz}$ ) para obtener información hidroacústica de la columna de agua y el fondo marino. El sistema hidroacústico fue calibrado cerca de la Isla San Lorenzo mediante el método de esferas propuesto por Foote et al. [1987]. Para la calibración se utilizó información de temperatura y salinidad de la columna de agua con la finalidad de determinar la velocidad del sonido promedio de la zona de trabajo. La calibración se realizó a una profundidad de 30 metros y se obtuvo una velocidad del sonido de $1525 \mathrm{~m} . \mathrm{s}^{-1}$ [Herbozo et al., 2016c]. Los valores de calibración fueron incluidos en el software Echoview [Higginbottom et al., 2008] para corregir la información hidroacústica adquirida durante el experimento de intercalibraciones. Una vez el experimento fue realizado y la información hidroacústica fue colectada, se eligieron ecogramas (que son matrices bidimensionales compuestas por información de Sv con profundidad de columna de agua y distancia GPS) de la zona superficial del fondo marino tomando en cuenta que el fondo marino se encuentre dentro de la plataforma continental y el relieve sea plano para evitar zonas escarpadas que influyan en la retrodispersión del eco. Fue necesario generar la línea de fondo y la línea de subfondo para establecer regiones de la zona superficial del fondo marino en base a $1 \mathrm{~m}$ de sedimento y $50 \mathrm{~m}$ de distancia GPS. Finalmente, se realizó la ecointegración de las regiones creadas para cada ecograma asociada a cada una de las 5 frecuencias disponibles. Adicionalmente, se compiló información sedimentológica recuperadas 
del fondo marino con la finalidad de realizar un análisis comparativo.

\section{Resultados}

\section{1 Área de estudio}

El área de estudio se encuentra al suroeste de Islas Hormigas, aproximadamente a 35 millas náuticas frente a Callao. Esta área se localiza entre la plataforma continental y el talud superior (profundidades entre $120 \mathrm{~m}$ y $1400 \mathrm{~m}$ de columna de agua). El transecto utilizado para exportar la información multifrecuencia tiene una distancia 7.5 $\mathrm{km}$ y se encuentra en una zona donde la morfología del suelo marino es plana, i.e., no presenta rasgos batimétricos con cambios abruptos de pendiente como escarpes ó altos estructurales. La profundidad promedio de columna de agua en este transecto es de $160 \mathrm{~m}$, la cual es registrada de igual forma en las cinco frecuencias investigadas.

\subsection{Muestras de sedimentos}

En términos generales, el área de estudio está caracterizada por un fondo marino compuesto por sedimentos fangosos en los primeros metros, información tomada mediante dragas y testigos de gravedad (e.g., testigo de caja) por Gutiérrez et al. [2006] y Reinhardt et al. [2002] (106KL). Sin embargo, en la plataforma continental a las afueras de Callao se han recuperado sedimentos superficiales compuesto por sedimentos fangosos y rocas pequeñas (e.g., $5 \mathrm{~cm}$ de diámetro) [Kudraß, 2002]. Adicional a lo recuperado por investigaciones previas, se han recuperado cortezas de fosforita al sureste de Islas Hormigas del fondo marino (con forma de discos y lozas) y dentro los primeros metros de sedimento fangoso superficial (con forma de nódulos densos y gravas) con espesores entre $5 \mathrm{~cm}$ y $10 \mathrm{~cm}$ [Arning et al., 2009]. A pesar de la compilación realizada de investigaciones previas, no encontramos muestreos realizados sobre el transecto prospectado. Por lo tanto, en este estudio no contamos con muestras del fondo marino superficial para realizar una comparación con la información

hidroacústica

multifrecuencia analizada.

\subsection{Información hidroacústica multifrecuencia}

En este estudio nos enfocamos en estudiar el primer metro por debajo del fondo marino. Por ende, el análisis realizado esta orientado a establecer patrones de reflectividad que permitan inferir los sustratos que componen la capa superficial del fondo marino. La información de retrodispersión acústica multifrecuencia obtenida a lo largo del transecto estudiado se presenta en las Figura 1. En la capa superficial (capa de $0 \mathrm{~m}$ a 1 $\mathrm{m}$, i.e., entre el relieve del fondo marino y 1 metro por debajo) definimos arbitrariamente patrones de reflectividad en base a la variabilidad del $S v$ mean por frecuencia que puedan describir sustratos específicos (e.g., fango laminado) en el fondo marino. Estos patrones han sido identificados como "p1" (km 44.4), "p2" (km 45.1), "p3" (km 45.4), "p4" (km 48.2), "p5" (km 49.7) y "p6" (km 50.9) (Figura 1). Los rangos de $S v$ mean para los patrones identificados según frecuencia se presentan en la Tabla 1. Al comparar las cinco frecuencias analizadas, tanto en el dominio logarítmico (Figuras 1a) como en el dominio lineal (Figuras 1b), se observa que los datos correspondientes a las frecuencias de $18 \mathrm{kHz}$ y $200 \mathrm{kHz}$ presentan valores extremos $(-2 \mathrm{~dB}$ y -32 $\mathrm{dB}$, Figura 1a) mientras que los datos de las frecuencias $38 \mathrm{kHz}, 70 \mathrm{kHz}$ y $120 \mathrm{kHz}$ presentan valores similares entre sí (de $-8 \mathrm{~dB}$ a $-22 \mathrm{~dB}$, Figura 1a). De los seis patrones definidos, tres de ellos (i.e., p1, p3, p6) presentan características distintivas. Estos patrones se encuentran mejor definidos en el dominio logarítimico (Figura 1a). En el caso de $\mathrm{p} 1$, existe una mayor reflectividad en las frecuencias de $200 \mathrm{kHz}$ y $18 \mathrm{kHz}$ comparada con p3. Los patrones p1 y p6 comparten valores similares en la frecuencia de $200 \mathrm{kHz}$. En el caso de $\mathrm{p} 3$, la reflectividad es menor en todas las frecuencias respecto de $\mathrm{p} 1$ y $\mathrm{p} 6$. Los tres patrones restantes (i.e., p2, p4, p5) son similares entre sí tanto en el dominio logarítmico como en el dominio lineal (Figura 1a y 1b). Existen diferencias en reflectividad en las frecuencias de $38 \mathrm{kHz}, 70 \mathrm{kHz}$ y $120 \mathrm{kHz}$. 

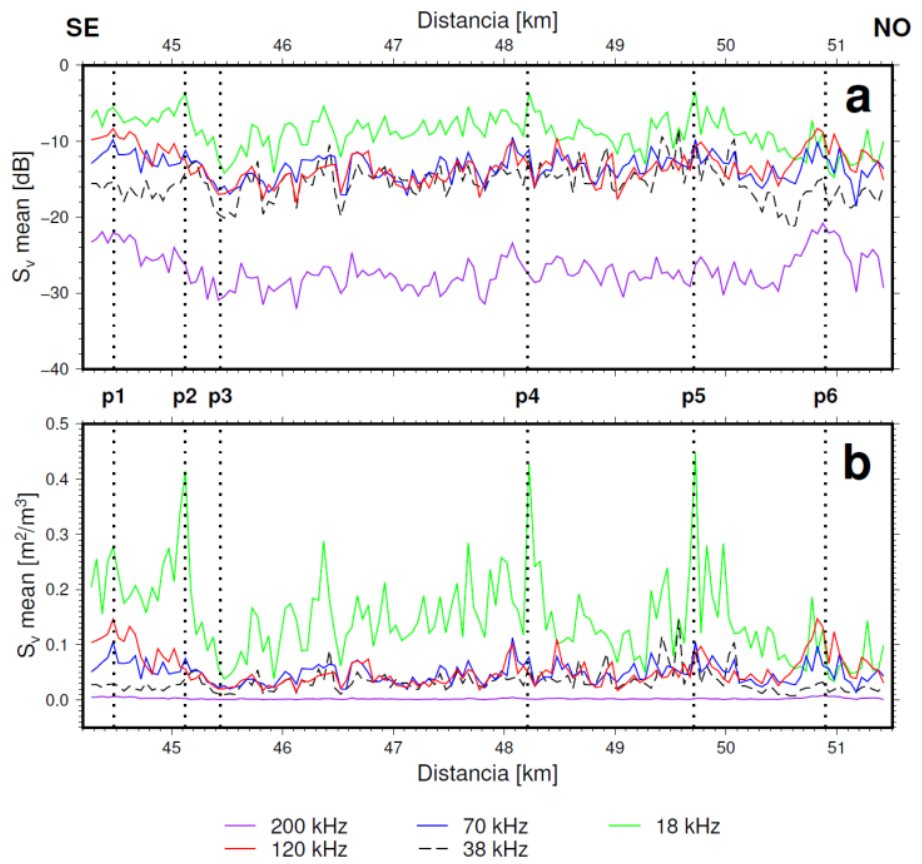

Figura 1: Información de retrodispersión acústica exportada por cada frecuencia según celdas de 50 $m$ de distancia GPS y $1 \mathrm{~m}$ por debajo del fondo marino: (a) en dominio logarítimo (dB), (b) en dominio lineal $\left(\mathrm{m}^{2} / \mathrm{m}^{3}\right)$. $p 1, p 2, p 3, p 4, \quad p 5=$ posiciones elegidas para realizar análisis comparativo de Sv mean.

Tabla 1: Comparación de reflectividad de patrones identificados para la capa superficial de sedimentos marinos (capa de 0 a $1 \mathrm{~m}$ ) según las 5 frecuencias utilizadas.

\begin{tabular}{|c|c|c|c|c|c|}
\hline \multirow{3}{*}{ Patrones } & \multicolumn{5}{|c|}{ Capa de 0 a $1 \mathrm{~m}$} \\
\hline & \multicolumn{5}{|c|}{ Frecuencias (kHz) } \\
\hline & 18 & 38 & 70 & 120 & 200 \\
\hline p1 (km 44.4) & $-5 \mathrm{~dB}$ & $-17 \mathrm{~dB}$ & $-11 \mathrm{~dB}$ & $-8.5 \mathrm{~dB}$ & $-23 \mathrm{~dB}$ \\
\hline $\mathrm{p} 2(\mathrm{~km} \mathrm{45.1)}$ & $-4 \mathrm{~dB}$ & $-14.8 \mathrm{~dB}$ & $-13 \mathrm{~dB}$ & $-13.5 \mathrm{~dB}$ & $-26 \mathrm{~dB}$ \\
\hline p3 (km 45.4) & $-14 \mathrm{~dB}$ & $-18.5 \mathrm{~dB}$ & $-16 \mathrm{~dB}$ & $-16.5 \mathrm{~dB}$ & $-30.5 \mathrm{~dB}$ \\
\hline $\mathrm{p} 4(\mathrm{~km} \mathrm{48.2)}$ & $-4 \mathrm{~dB}$ & $-13 \mathrm{~dB}$ & $-11 \mathrm{~dB}$ & $-12 \mathrm{~dB}$ & $-18 \mathrm{~dB}$ \\
\hline p5 (km 49.7) & $-4 \mathrm{~dB}$ & $-10 \mathrm{~dB}$ & $-9 \mathrm{~dB}$ & $-11 d b$ & $-25 \mathrm{~dB}$ \\
\hline p6 (km 50.5) & $-9 \mathrm{~dB}$ & $-15 \mathrm{~dB}$ & $-12 \mathrm{~dB}$ & $-9 \mathrm{~dB}$ & $-21 \mathrm{~dB}$ \\
\hline
\end{tabular}

\section{Discusión}

\subsection{Exploración de sustratos según reflecti- vidad}

En el transecto analizado no encontramos información sedimentológica del primer metro por debajo del fondo marino, por ende no es posible calibrar la reflectividad acústica derivada en este estudio para definir una clasificación comparativa (i.e., muestras de sedimento versus reflectividad).
Sin embargo, podemos estimar la composición general de los sustratos de esta capa superficial en base a las frecuencias empleadas. Esta diferenciación se basa en el principio de que cada sustrato presenta una impedancia acústica específica, por ende una resistencia al paso del sonido; lo cual promueve la atenuación del sonido emitido, su respectiva reflexión (ó reflectividad) y transmisión en el sedimento. Este proceso físico es análogo al de aventar una pelota (e.g., de tenis) con la misma fuerza contra dos superficies, una pared de concreto y un colchón de dormir. En el caso de la pared de concreto, la pelota rebotará más lejos con mayor fuerza mientras que en el caso del colchón la misma pelota rebotará a menor distancia y menor fuerza (i.e., el resultado es directamente proporcional a la característica de la superficie). Tal fenómeno se explica principalmente por la mayor densidad del concreto respecto de la del colchón. En el caso de los sedimentos marinos superficiales investigados, este principio funciona de forma similar, i.e., sedimentos más densos generarán una mayor reflectividad mientras que sedimentos menos densos serán más permeables al paso del sonido, por ende presentarán una menor reflectividad (i.e., existirá una mayor transmisión del sonido en el sustrato). Existe un proceso adicional que es clave para entender la reflectividad de los sustratos, el cual se le denomina resonancia. Este proceso se relaciona a la interacción entre la longitud de onda (i.e., frecuencia) y el tamaño de las partículas de los materiales investigados, por ende capacidad de resolución vertical de los datos y la reflectividad del sonido emitido. Usando los conceptos descritos previamente descritos, podemos interpretar la relación que existe entre las reflectividades derivada de la primera capa superficial del fondo marino ploteada en la Figura 1. Los patrones p2, p4 y p5 tienen reflectividades similares, lo cual sugiere que los sedimentos superficiales en estas zonas, aproximadamente entre $50 \mathrm{~m}$ y $100 \mathrm{~m}$ de longitud a lo largo del transecto, presentan una composición similar. Estos patrones presentan una alta reflectividad para la frecuencia de $18 \mathrm{kHz}$, reflectividades similares para $38 \mathrm{kHz}, 70 \mathrm{kHz}$ y 120 $\mathrm{kHz}$, y una menor reflectividad para $200 \mathrm{kHz}$. Nosotros interpretamos que estos patrones representan sedimentos compuestos por sustratos 
relativamente duros próximos al fondo marino (e.g., lozas de fosforita) con una mezcla de sedimentos fangosos arenosos por debajo de estos sustratos duros superficiales. Esta interpretación se basa en la presencia de materiales densos que son resonantes a una frecuencia particular (e.g., 18 $\mathrm{kHz}$ ) lo que genera una mayor reflectividad, que a su vez promueven una mayor atenuación respecto de frecuencias más altas (e.g., 200 kHz). Se conoce que en la zona de Callao existen lozas y cortezas de fosforita sobre el fondo marino y en los primeros metros debajo del fondo marino con varios espesores y asociado a sedimentos finos [Suess and von Huene, 1988; Arning et al., 2009], lo cual apoya nuestra interpretación para p2, p4 y p5. En la zona del p3 se observa que las reflectividades son menores respecto de los demás patrones (Figura 1). Nuestra interpretación es que la zona de p3 (100 m-200m a lo largo del transecto) esta compuesta por sedimentos finos (e.g., fango laminado), probablemente asociado a los mud lens de acuerdo a las bajas reflectividades observadas, por ende mayor transmisión del sonido en el sedimento. Respecto del p1 y p6, se observan altas reflectividades con excepción de la frecuencia de $18 \mathrm{kHz}$ y $38 \mathrm{kHz}$. Las reflectividades son especialmente altas en las frecuencias más altas que son la de $200 \mathrm{kHz}, 120 \mathrm{kHz}$ y $70 \mathrm{kHz}$ (i.e., menor longitud de onda). Para estos patrones sugerimos que tal respuesta acústica se debe a la presencia de frentes de gas que generan una alta reflectividad por el gran contraste de impedancia acústica producto del gas natural y la resonancia de las burbujas para la frecuencias más altas, por ende la atenuación en profundidad si el frente de gas no es lo suficientemente profundo [e.g., Judd and Hovland, 1992; Baltzer et al., 2005]. Nosotros sugerimos que el gas presente es gas metano de origen biogénico debido a la descomposición de materia orgánica depositada producto de la alta productividad biológica en el mar Peruano [Suess and von Huene, 1988; Pennington et al., 2006]. Estas zonas cubrirían aproximadamente 500 metros a lo largo del transecto investigado tanto en la zona de $\mathrm{p} 1$ como en la zona de $\mathrm{p} 6$.

El siguiente paso para extender los resultados e interpretaciones de esta investigación es la validación de las reflectividades investigadas con muestras in-situ de las zonas prospectadas con hidroacústica. Adicionalmente, es necesario cuantificar la fluctuación asociada a la reflectividad del múltiplo del fondo marino, dado que la reflectividad de este reflector es proporcional a la reflectividad del fondo marino. La validación de la información hidroacústica permitirá una clasificación remota de amplias zonas del fondo marino en un corto tiempo. Desde el punto de vista de recursos pesqueros, tal definición de tipos de fondos integrado con cartas de distribución de especies demersales como la merluza peruana, permitirá conocer si existe una influencia del tipo de sustrato superficial en el fondo marino con la abundancia de estas especies. Otro tipo de organismo que tiene relación con el tipo de fondo es la Thioploca. Las comunidades de esta bacteria filamentosa se encuentran distribuidas en forma de matas cubriendo el fondo marino y asociadas a sedimentos fangosos dentro de la ZMO en la plataforma y talud continental en la zona central del margen peruano [Gutiérrez et al., 2006]. Por lo tanto, al conocer la distribución de los tipos de sedimentos de forma remota se podría caracterizar la distribución de esta bacteria gigante que es clave para el estudio de la biogeoquímica de los procesos de afloramiento, la extensión vertical de la ZMO, la interfase agua-sedimento y la degradación de materia orgánica presente en el fondo marino. La clasificación remota del tipo de fondo también sería clave para conocer áreas propicias para el desarrollo de acuicultura, lo cual es un tema necesario para estudios de impacto ambiental relacionados con esta actividad económica. Los puntos anteriores se relacionan con el estudio del fondo marino, sin embargo, la metodología y análisis de la reflectividad multifrecuencia tiene la potencialidad de proveer información para estimar la composición de cardúmenes de especies pelágicas como la anchoveta (e.g., en base a porcentaje de juveniles y adultos respecto del total del cardumen) [Herbozo et al., en prep.]. Esta caracterización puede proporcionar índices iniciales para poder diferenciar estos cardúmenes y/o agregaciones de anchoveta, lo cual sería importante para la captura específica de cardúmenes compuestos de adultos y la preservación de cardúmenes de juveniles. Por ende, tal análisis tendría una aplicación directa en la cuantificación de biomasa de esta especie 
pelágica, donde su comportamiento y distribución dependen de la variabilidad semanal de las condiciones oceanográficas en el mar Peruano [Herbozo et al., 2016a].

\section{Conclusiones}

Mediante la presente investigación estudiamos, en base a información hidroacústica multifrecuencia de una ecosonda científica del IMARPE, la composición general del primer metro de sedimentos marinos localizados al suroeste de las Islas Hormigas de Afuera, costa afuera de Callao. En este estudio no se pudo comparar la reflectividad acústica de esta capa superficial con muestras de sedimentos recuperadas del fondo marino en la zona de estudio. De la reflectividad analizada, se observó que existen patrones que permitirían realizar una estimación de la composición sedimentaria. La reflectividad en tres patrones elegidos (p2, p4 y p5) podrían representar zonas donde existen sustratos duros cerca al fondo marino (e.g., cortezas de fosforita y/o rocas del orden de $\mathrm{cm}$ de espesor) y por debajo sedimentos finos y gruesos (e.g., fangos y arenas gruesas). Debido a la permeabilidad de la capa superficial, un patrón (p3) podría representar sedimentos finos probablemente asociados al mud lens. Este tipo de depocentros sedimentarios estan compuestos por sedimentos finamente laminados y que son acústicamente permeables como las arcillas finas y/o una intercalación de sustratos finos. Los dos patrones localizados a los extremos del transecto ( $\mathrm{p} 1$ y $\mathrm{p} 6$ ) podrían representar zonas donde existen frentes de gas natural distribuidos en sedimento fangoso.

\section{Agradecimientos}

Agradecimientos a la Dirección General de Investigaciones de Hidroacústica, Sensoramiento Remoto y Artes de Pesca del IMARPE por las facilidades brindadas para el desarrollo del presente informe. El análisis espacial y figuras fueron creadas con software de código libre (también conocido como software libre) (e.g., Generic Mapping Tools (GMT) [Wessel et al., 2013]).

\section{Referencias}

[1] Andersen, L. N. [2001], The new SIMRAD EK60 scientific echo sounder system, The Journal of the Acoustical Society of America 109(5), 2336-2336.

[2] Arning, E., Lückge, A., Breuer, C., Gussone, N., Birgel, D. and Peckmann, J. [2009], Genesis of phosphorite crusts off Peru, Marine Geology 262(1), 68-81.

[3] Böning, P., Brumsack, H.-J., Böttcher, M. E., Schnetger, B., Kriete, C., Kallmeyer, J. and Borchers, S. L. [2004], Geochemistry of Peruvian near-surface sediments, Geochimica et Cosmochimica Acta 68(21), 4429-4451.

[4] Cuff, A., Anderson, J. T. and Devillers, R. [2015], Comparing surficial sediments maps interpreted by experts with dualfrequency acoustic backscatter on the Scotian Shelf, Canada, Continental Shelf Research 110, 149-161.

[5] D'Hondt, S., Joergensen, B. and Miller, D. [2003], ODP Leg 201 Scientific Party: Controls on microbial communities in deeply buried sediment, Eastern Equatorial Pacific and Peru Margin, sites 1225-1231, in 'Proceedings ODP, Initial Reports', Vol. 201.

[6] Foote, K., Knudsen, H., Vestnes, G., Maclennan, D. and Simmonds, E. [1987a], Calibration of acoustic instruments for fish density estimation: a practical guide, 144, 69.

[7] Gutiérrez, D., Sifeddine, A., Field, D., Ortlieb, L., Vargas, G., Chávez, F., Velazco, F., Ferreira, V., Tapia, P., Salvatteci, R. et al. [2009], Rapid reorganization in ocean biogeochemistry off Peru towards the end of the Little Ice Age, Biogeosciences 6, 835848.

[8] Gutiérrez, D., Sifeddine, A., Reyss, J., Vargas, G., Velazco, F., Salvatteci, R., Ferreira, V., Ortlieb, L., Field, D., Baumgartner,

T. et al. [2006], Anoxic sediments off Central Peru record interannual to multidecadal changes of climate and upwelling ecosystem during the last two centuries, Advances in Geosciences 6, 119-125.

[9] Gutiérrez, M. and Herbozo, G. [2004], 
Batimetría y clasificación exploratoria del fondo marino utilizando información de retrodispersión acústica, Instituto del Mar del Perú (IMARPE). Informe Acústico 0405 Paleoceanografía, Julio 2004, Unidad de Tectonología de Detección.

[10] Herbozo, G., Fuertes, J., Santivañez, M., La Cruz, L. and Calderón, J. [2016b], Estimación inicial de incertidumbre en las estimaciones hidroacústicas de biomasa de la Anchoveta Peruana durante los últimos 26 años (1990-2015), Instituto del Mar del Perú (IMARPE). Área Funcional de Hidroacústica (AFH), Dirección General de Investigaciones en Hidroacústica, Sensoramiento Remoto y Artes de Pesca (DGIHSA).

[11] Herbozo, G., Santivañez, M., Fuertes, J., La Cruz, L. and Pablo, A. [2016c], Experimento de intercalibraciones entre el BIC José Olaya Balandra del IMARPE y buques pesqueros de la flota industrial, Instituto del Mar del Perú (IMARPE). Área Funcional de Hidroacústica (AFH), Dirección General de Investigaciones en Hidroacústica, Sensoramiento Remoto y Artes de Pesca (DGIHSA).

[12] Herbozo, G., Fuertes, J., Santivañez, M., and La Cruz, L. [en preparación]. Patrones de clasificación exploratoria de cardúmenes de anchoveta mediante análisis multifrecuencia, Instituto del Mar del Perú (IMARPE). Área Funcional de Hidroacústica (AFH), Dirección General de Investigaciones en Hidroacústica, Sensoramiento Remoto y Artes de Pesca (DGIHSA).

[13] Higginbottom, I., Woon, S., Schneider, P. [2008], Hydroacoustic data processing for standard stock assessment using Echoview: technical manual. FAO/ADRIAMED.

[14] Kudraß, H. [2002], Abschlußbericht PeruAuftrieb, SONNE-Fahrt SO147 Final Report:(BMBF Forschungsvorhaben 03G 0147 A).

[15] Morón, O. [2000], Características del ambiente marino frente a la costa peruana, Boletín del Instituto del Mar del Perú 19(1-2), 179-204.

[16] Rein, B., Lückge, A., Reinhardt, L., Sirocko, F., Wolf, A. and Dullo, W.-C. [2005], El Niño variability off Peru during the last 20,000 years, Paleoceanography 20(4).

[17] Reinhardt, L., Kudrass, H.-R., Lückge, A., Wiedicke, M., Wunderlich, J. and Wendt, G. [2002], High-resolution sediment echosounding off Peru: Late Quaternary depositional sequences and sedimentary structures of a current-dominated shelf, Marine Geophysical Researchers 23(4), 335-351.

[18] Salvatteci, R., Field, D., Sifeddine, A., Ortlieb, L., Ferreira, V., Baumgartner, T., Caquineau, S., Velazco, F., Reyss, J.-L., Sanchez-Cabeza, J.-A. et al. [2014], Crossstratigraphies from a seismically active mud lens off Peru indicate horizontal extensions of laminae, missing sequences, and a need for multiple cores for high resolution records, Marine Geology 357, 72-89.

[19] Salvatteci, R., Gutiérrez, D., Field, D., Sifeddine, A., Ortlieb, L., Bouloubassi, I., Boussafir, M., Boucher, $H$. and Cetin, F. [2013], The response of the Peruvian Upwelling Ecosystem to centennial-scale global change during the last two millennia, Climate of the Past Discussions 9, 54795519.

[20] Stramma, L., Johnson, G. C., Sprintall, J. and Mohrholz, V. [2008], Expanding oxygenminimum zones in the tropical oceans, science 320(5876), 655-658.

[21] Suess, E. and von Huene, R. [1988], ODP Leg 112 Scientific Party: Peru continental margin, sites 679-688, in 'Proceedings ODP, Initial Reports', Vol. 112.

[22] Wessel, P., Smith, W. H., Scharroo, R., Luis, J. and Wobbe, F. [2013], Generic Mapping Tools: Improved Version Released, Eos, Transactions American Geophysical Union 94(45), 409-410.

E-mails:geryherbozo@gmail.com, ifuertesc171990@gmail.com,dmamani@imarpe.go b.pe, fvelazco@imarpe.gob.pe 